\section{Weniger stationäre Behandlungen durch Rotavirus-Impfung}

- Die Aufnahme der Rotavirus-Impfung für Säuglinge in nationale Impfpläne kann die Rate der virusbedingten stationären Behandlungen schnell und stark verringern. Das zeigen Untersuchungen aus verschiedenen Ländern, berichtete PD Dr. Markus Rose aus Frankfurt am Main. Wichtig ist die Impfung vor allem deshalb, weil schwere Gastroenteritiden bei Kleinkindern und Säuglingen meist durch eine Rotavirus-Infektion entstehen. Sie führt in Deutschland jährlich zu rund 22.000 Klinikaufnahmen von Kindern unter fünf Jahren.

Dagegen minderte die landesweite Impfung beispielweise in Österreich die Rate rotavirusbedingter Klinikaufenthalte um $42 \%$ (Kinder im Alter unter 90 Tagen) und 20 Monate). Dieser Erfolg wurde innerhalb von nur eineinhalb Jahren nach Einführen des Impfprogramms erzielt. Die Impfrate betrug zu diesem Zeitpunkt schon $87 \%$. Daten anderer Länder bestätigen die Wirksamkeit. So wurde in Queensland/Australien seit 2007 die Wirkung des Schluckimpfstoffs RotaTeq ${ }^{\circledR}$ notiert. Er wirkt gegen fünf Serotypen, die 98\% der Rotavirus-Infektionen in Europa verantworten. Die Serotypen-Verteilung in Australien ist ähnlich. Mit der Impfung ging die Zahl rotavirusbedingter Gastroenteritiden bis zum Jahr 2008 bei Kindern unter zwei Jahren um $65 \%$ zurück. Eine neue Studie aus Frankreich bestätigt dieses Ergebnis: Hospitalisierungen waren bei un- um 73\% (Kinder im Alter von 90 Tage bis ter 2-jährigen Kindern nach nur einem Jahr nur noch halb so häufig wie erwartet. zwei Jahre nach Einführen der Impfung bei Kindern unter drei Jahren deutlich weniger Klinikaufenthalte durch Rotavirus-Infektionen. In New York etwa sank die Zahl um $85 \%$. Außerdem entwickelt sich offenbar eine Herdenimmunität, wie Rose berichtete. Denn auch nicht geimpfte, ältere Kinder erkrankten seltener als früher. Der Nutzen der Impfung ist also deutlich. Trotzdem existiert hierzulande erst in einigen Bundesländern, etwa Sachsen, eine generelle Rotavirus-Impfempfehlung.

HBR

Pressekonferenz „Rotavirus-Schluckimpfung: Erfolgreiche Umsetzung, Wirksamkeit und Sicherheit durch aktuelle Daten belegt“. Frankfurt/Main, 2. März 2010. Veranstalter: Sanofi Pasteur MSD, Leimen In den USA wiederum verzeichnete man

\section{Asthma-Kontrolle mit wenig Steroid}

— Weniger als die Hälfte der Kinder mit Asthmakontrolle wird nach Meinung von Prof. Monika Gappa aus Wesel immer noch viel zu selten erreicht. Dabei, so Gappa, sind die Pädiater anspruchsvoll. Für sie heißt Asthmakontrolle ein Leben, bei dem die Kinder nicht merken, dass sie an einer chronischen Atemwegserkrankung leiden. Die Therapie als Asthmakontrolle hat dabei die früher übliche Stufentherapie nach Schweregrad abgelöst. Einbezogen wird der Asthmakontrolltest, in dem Eltern und Kinder über die asthmabezogenen Beschwerden befragt werden. Die wenigen Fragen geben Auskunft, ob Maßnahmen zur Therapieoptimierung nötig sind.

Die Ursachen für die hohe Zahl unzureichend kontrollierter Patienten liegt oft an einer Falscheinschätzung durch den Patienten und einer schlechten Compliance. Viele Patienten haben Angst vor dem Wirkstoff Kortison. Durch die Fixkombination aus dem inhalativen Kortikosteroid Fluticason und dem langwirksamen Beta-Agonisten Salmeterol (Viani ${ }^{\circledR}$ Diskus $^{\circledR}$ ) kann die Compliance verbessert werden.

Dass auch bei jungen Patienten mit dieser Fixkombination eine gut Asthmakontrolle zu erreichen ist, bestätigen aktuelle Studiendaten aus Deutschland. Im Vergleich mit Asthma wird richtig behandelt. Eine gute der doppelten Fluticason-Dosis erwies sich die Fixkombination Salmeterol/Fluticason als mindestens genauso wirksam und hatte darüber hinaus einen steroidsparenden Effekt. Die Patienten verbrachten signifikant weniger Tage ohne Bedarfsmedikation und ihre Symptome verbesserten sich im Vergleich zur Monotherapie. Kontrolliert ist Asthma nach GINA-Kriterien, wenn tagsüber höchstens zweimal pro Woche und nachts nie Symptome auftreten, keine Aktivitätseinschränkungen bestehen, höchstens zweimal pro Woche Bedarfsmedikation benötigt wird, die Lungenfunktion normal ist und keine Exazerbationen auftreten.

Pressekonferenz „Unbeschwertes LEBEN durch GINA-definierte Asthmakontrolle“. Nettetal, 9. Dezember 2009. Veranstalter: GlaxoSmithKline, München

\section{HPV-Impfung in Deutschland rückläufig}

Wegen niedriger Impfraten gegen humane Papillomaviren (HPV) ist das Ziel in Gefahr, einen Rückgang an Gebärmutterhalskrebs zu erreichen. Lag in Deutschland die Impfrate bei 12- bis 17-jährigen Mädchen im Juli 2008 noch bei $38 \%$, so war sie im Juli 2009 auf 33\% abgesunken.

Zwar lässt sich laut Prof. Monika Hampl, Düsseldorf, eine Verringerung der Inzidenz an Gebärmutterhalskrebs erst nach Jahrzehnten messen, aber erste Effekte der Impfung sind schon jetzt nachweisbar. Dies zeigt eine australische retrospektive Beobachtungsstudie von Fairley et al.: Seit 2007 wird bei 12- bis 18-jährigen Mädchen eine Impfung mit Gardasil ${ }^{\circledR}$ in der Schule und bei unter 26-jährigen Frauen die Impfung in Praxen angeboten. Ein Jahr nach Impfbeginn und bei einer Durchimpfungsrate von ca. 70\% ging der Anteil an Genitalwarzen bei den Frauen um 48\% zurück.

Auch andere Studien zu Gardasil ${ }^{\circledR}$ zeigten erste Erfolge. Beispielsweise wurden in umfangreichen Zulassungsstudien 17.622 Frauen im Alter zwischen 16 und 26 Jahren über dreieinhalb Jahre untersucht. Gegenüber der Kontrollgruppe traten bei den geimpften Frauen 45\% weniger hochgradige intraepitheliale Läsionen auf.

Pressegespräch „HPV-Impfung in Deutschland: Präventionserfolg in Gefahr“. Frankfurt, 4. November 2009. Veranstalter: Sanofi Pasteur MSD, Leimen 